\title{
Maternal 75-g OGTT glucose levels as predictive factors for large-for- gestational age newborns in women with gestational diabetes mellitus
}

Krstevska Brankica', Velkoska Nakova Valentina², Simeonova Krstevska Slagjana ${ }^{3}$, Jovanovska Mishevska Sasha'

1 Endocrinology Diabetes and Metabolic Disorders Clinic, Medical Faculty, Skopje, R. Macedonia ${ }^{2}$ Faculty of Medical Science, Goce Delcev University, Stip, R. Macedonia ${ }^{3}$ Gynecology and Obstetric Clinic, Medical Faculty, Skopje, R. Macedonia

\section{Correspondence to:}

Krstevska Brankica

Endocrinology Diabetes and Metabolic Disorders Clinic,

Medical Faculty,

Skopje, R. Macedonia

1000 - Skopje, R. Macedonia branakrstevska@yahoo.com

Received on Aug/16/2013 Accepted on Sept/29/2015

DOI: 10.1590/2359-3997000000126

\begin{abstract}
Objective: Our goal was to investigate which glucose measurement from the 75-g oral glucose tolerance test (OGTT) has more capability of predicting large for-gestational-age (LGA) newborns of mothers with gestational diabetes mellitus (GDM). Subjects and methods: The study group consisted of 118 consecutively pregnant women with singleton pregnancy, patients of Outpatients Department of the Endocrinology, Diabetes, and Metabolic Disorders Clinic. All were prospectively screened for GDM between $24^{\text {th }}$ and $28^{\text {th }}$ week of pregnancy and followed to delivery. Outcome measures included: patients' ages, pre-pregnancy BMI, BMI before delivery, FPG, 1 and 2 hour OGTT glucose values, haemoglobin $\mathrm{A} 1 \mathrm{c}$ at third trimester, gestational week of delivery, mode of delivery and baby birth weight. Results: From 118 pregnancies, $78(66.1 \%)$ women were with GDM, and 40 (33.9\%) without GDM. There were statistically significant differences $(30.7$ versus $5.0 \%, p<0.01)$ between LGA newborns from GDM and control group, respectively. Gestation week of delivery and fasting glucose levels were independent predictors for LGA (Beta $=0.58$ and Beta $=0.37$ respectively, $p<0.01$ ). Areas under the receiver operator characteristic curve (AUC) were compared for the prediction of LGA (0.782 (0.685-0.861) for fasting, $0.719(0.607-0.815)$ for 1 -hour and $0.51(0.392-0.626)$ for 2 -hour OGTT plasma glucose levels). Conclusion: Fasting and 1-hour plasma glucose levels from OGTT may predict LGA babies in GDM pregnancies. Arch Endocrinol Metab. 2016;60(1):36-41
\end{abstract}

Keywords

Gestational diabetes; oral glucose tolerance test (OGTT); large for gestational age

\section{INTRODUCTION}

$\mathrm{G}$ estational diabetes mellitus (GDM) is a common I medical condition defined as a carbohydrate intolerance that begins or is first diagnosed during pregnancy (1). Maternal supply of carbohydrates leads to fetal hyperglycaemia, which in turn stimulates fetal pancreatic islet cells and causes hyperinsulinaemia (2). Stimulation of the insulin-sensitive tissue results in increased fetal growth, predominantly of the abdomen, and delivery of large for gestational-age (LGA) newborns (3). Both women with large fetuses and LGA newborns are at a higher risk of complications (4).

Oral glucose tolerance test (OGTT) is accepted as a diagnostic "gold standard" for GDM diagnosis. The National Diabetes Data Group (NDDG) first introduced the 3-h 100-g OGTT test as diagnostic test for GDM. This test has ability to predict postpartum diabetes mellitus in women with GDM. Later, the WHO adopted the 2-h 75-g OGTT recommending the same diagnostic cut points established for the diagnosis of impaired glucose tolerance outside of pregnancy. In 1999, WHO clarified that GDM encompassed impaired glucose tolerance and diabetes (fasting $\geq 7 \mathrm{mmol} / \mathrm{L}, 2$-h $\geq 7.8 \mathrm{mmol} / \mathrm{L}$ ) and over the years has maintained their recommendations (5). The recently published Hyperglycemia and Adverse Pregnancy Outcomes (HAPO) study confirmed the link between hyperglycemia and adverse pregnancy outcomes, using the $2 \mathrm{~h} 75$-g OGTT with new cut points: FPG $\geq 5.1 \mathrm{mmol} / \mathrm{L}, \mathrm{l}-\mathrm{h} \geq 10.0$ $\mathrm{mmol} / \mathrm{L}, 2-\mathrm{h} \geq 8.5 \mathrm{mmol} / \mathrm{L}(6)$. In response to these results, the International Association of the Diabetes in Pregnancy Study Group (IADPSG) and American Diabetes Association (ADA) formulated new guidelines for screening and diagnosis of GDM (7).

There is no doubt from the literature that maternal glycaemia in women with GDM is involved in determi- 
ning birth weight, but whether there is any influence of maternal glycaemia during OGTT on fetal over growth is unknown $(8)$.

Black and cols. recently demonstrated that the risk of adverse pregnancy outcomes differs between women with impaired fasting plasma glucose values (FPG) and abnormal glucose levels during the OGTT, providing evidence that women with elevated FPG particularly suffer from delivering LGA infants (9). Ferrara and cols. found that both maternal FPG and $1-h$, but not 2 or 3-h from 100-g. OGTT glucose levels were independent predictors of macrosomic risk (10). Caulfield and cols. evaluated risk of macrosomia according to abnormal glucose results and found that risk for infant macrosomia was higher among women with GDM only if they had fasting hyperglycemia (11). But, Kösüs and cols. found that 2 and $3 \mathrm{~h}$ glucose levels were the most important predictors of the macrosomia (12).

Excess nutrient delivery to the fetus causes LGA newborns, but whether fasting or peak glucose levels are more correlated with fetal overgrowth is less clear. So, we decided to explore the relation between the results of 2-h 75-g OGTT and neonatal birth weight.

The aim of this study was to investigate which glucose measurement from the 75-g OGTT (FPG, l-h, and 2 -h) has more capability of predicting large for-gestational-age (LGA) newborns of mothers with GDM.

\section{SUBJECTS AND METHODS}

One hundred and eighteen pregnant women were prospectively screened for GDM between $24^{\text {th }}$ and $28^{\text {th }}$ week of pregnancy. The pregnant women were Outpatients of the Endocrinology, Diabetes, and Metabolic Disorders Department of the University Clinical Hospital in Skopje, R. Macedonia in a period from 01.2013 to 06.2013 . GDM was defined according to the IADPSG (7), criteria for glucose measurements during 2-hours 75-g OGTT, as at least one level greater than fasting plasma glucose equal to or exceeding 5.1 $\mathrm{mmol} / \mathrm{L}, 1$ hour OGTT glucose equal to or exceeding $10.0 \mathrm{mmol} / \mathrm{L}$, and 2 hour OGTT glucose equal to or exceeding $8.5 \mathrm{mmol} / \mathrm{L}$. We followed participants until delivery and obtained delivery outcome data.

The following parameters were analyzed: patients' ages, pre-pregnancy body mass index (BMI), BMI before delivery, FPG, 1 and 2 hour OGTT glucose values, haemoglobin $\mathrm{Alc}(\mathrm{HbAlc})$ at third trimester, gestational week of delivery, mode of delivery and baby birth weight.
All of the patients underwent 75-g OGTT, following the standard recommendations (13). Briefly, 75-g a hydrous glucose load was administered after a 12- to 14-h fast and fasting, 1-h post-load and 2-h post-load samples for glucose were obtained from an antecubital vein. Samples were collected in tubes containing fluoride and kept at $4^{\circ} \mathrm{C}$ until centrifugation up to $2 \mathrm{~h}$ later. Plasma measurements were performed with glucose oxidase methods.

The BMI of women was calculated by dividing the weights by the squared heights in meters $\left(\mathrm{km}^{2}\right)$. The patients were weighed wearing clothes without shoes in the morning with an electronic scale at the first visit. Height was measured to the nearest $\mathrm{l} \mathrm{cm}$ with a stadiometer. At enrollment pre-pregnancy BMI was calculated by using the most recent self reported weight before conception. Blood samples for HbAlc were taken after overnight fasting. HbAlc was measured by an ion-exchange HPLC instrument (DS5; Drew, USA) with a reference range of $4.2-6 \%$. We followed participants until delivery. Neonatal outcomes were performed in University Clinic of Gynecology and Obstetrics. Mode of delivery was noted as spontaneous or caesarean section. Birth weight and proportion of LGA (defined as a birth weight $>90^{\text {th }}$ percentile for local population after adjusting for gestational age and sex) were determined. The gestational age of newborns was estimated from the date of the last menstrual period.

All patients gave informed consent to participate in the study after receiving explanations regarding the research protocol. The study was done according to the Helsinki Declaration.

\section{Statistical analyses}

Statistical analyses were performed by SPSS 14.0 software. The significance of the differences between pregnant women with and without GDM was tested using $\mathrm{t}$-test for numeric variables, and $\chi^{2}$-test for categorical variables. To determine the relationship between analyzed variables Spearman's correlation test was used. In regression model all variables were grouped into one block and were entered in a single step using "enter" regression method. The variables with no collinearity $(\mathrm{r}<0.70)$ were considered for further analyses. Coolinearity between the independent variables was assessed pair-wise by calculation of Spearman rang correlations. As goodness-of-fit statistics was used $\mathrm{R}$ square change if the $\mathrm{R}^{2}$ change associated with a variable is large, that means that variable is a good predictor of the dependent variable. LGA was dependent variable. We plotted 
receiver operator characteristic (ROC) curves for predicting LGA newborns, and estimated and compared the area under the curve (AUC) using the MedCalc Statistical Software, version 12.5.0. Differences were considered significant if $\mathrm{p}$ was less than 0.05 .

\section{RESULTS}

From 118 included women, $78(66.1 \%)$ were with GDM. The baseline data of all included pregnant women are presented in Table 1.

As expected, women with GDM were older, more overweight before pregnancy, and with higher weight gain than women with normal glucose tolerance, but the differences were slight, so they did not reach statistical significance. There were statistically significant differences between proportions of LGA newborns from GDM and normal pregnancies. The incidence of cesarean section was not different between the women with GDM and women without GDM (Table 1).

Table 1. Baseline characteristics of all included pregnant women

\begin{tabular}{lccc}
\hline & $\begin{array}{c}\text { GDM } \\
(\mathbf{n = 7 8})\end{array}$ & $\begin{array}{c}\text { Without GDM } \\
(\mathbf{n = 4 0 )}\end{array}$ & $\boldsymbol{p}$ value \\
\hline Age (years) & $32.6 \pm 4.8$ & $31.4 \pm 5.1$ & $\mathrm{NS}$ \\
Pre-pregnancy BMI $\left(\mathrm{kg} / \mathrm{m}^{2}\right)$ & $26.4 \pm 6.3$ & $25.9 \pm 4.4$ & $\mathrm{NS}$ \\
BMl before delivery $\left(\mathrm{kg} / \mathrm{m}^{2}\right)$ & $\mathbf{3 0 . 1} \pm \mathbf{4 . 9}$ & $\mathbf{2 6 . 5} \pm \mathbf{8 . 9}$ & $<\mathbf{0 . 0 1}$ \\
HbA1c (\%) & $\mathbf{5 . 2} \pm \mathbf{1 . 2}$ & $\mathbf{4 . 7} \pm \mathbf{0 . 2}$ & $<\mathbf{0 . 0 1}$ \\
FPG (mmol/L) & $\mathbf{5 . 8} \pm \mathbf{1 . 2}$ & $\mathbf{4 . 7} \pm \mathbf{0 . 5}$ & $<\mathbf{0 . 0 1}$ \\
1-hour OGTT (mmol/L) & $\mathbf{1 1 . 3} \pm \mathbf{1 . 8}$ & $\mathbf{7 . 4} \pm \mathbf{1 . 4}$ & $<\mathbf{0 . 0 1}$ \\
2-hour OGTT (mmol/L) & $\mathbf{9 . 3} \pm \mathbf{2 . 1}$ & $\mathbf{6 . 2} \pm \mathbf{1 . 2}$ & $<\mathbf{0 . 0 1}$ \\
Birth weight (g.) & $\mathbf{3 , 5 6 9} \pm \mathbf{6 8 1}$ & $\mathbf{3 , 2 6 7} \pm \mathbf{5 0 1}$ & $<\mathbf{0 . 0 5}$ \\
LGA (\%) & $\mathbf{2 4 / 7 8 ~ ( 3 0 . 7 )}$ & $\mathbf{2 / 4 0 ~ ( 5 . 0 )}$ & $<\mathbf{0 . 0 1}$ \\
Cesarean section (\%) & $37 / 78(47.4 \%)$ & $9 / 40(22.5 \%)$ & NS \\
Gestational week of & $38.8 \pm 1.8$ & $39.3 \pm 1.3$ & NS \\
delivery & & & \\
\hline
\end{tabular}

Displayed results are average \pm std deviation and percentages. Comparisons between the 2 groups were performed by student's t-test for continuous variables and $\chi^{2}$-test for categorical variables.

BMl: body mass index; HbA1c: glycosylated haemoglobin; FPG: fasting plasma glucose; OGTT: oral glucose tolerance test; LGA: large for gestational age; NS: no significance.

There were significant correlations between LGA newborns from GDM pregnancies with BMI before pregnancy $(\mathrm{r}=0.36, \mathrm{p}<0.01)$, BMI before delivery $(\mathrm{r}=0.36, \mathrm{p}<0.01)$, FPG $(\mathrm{r}=0.46, \mathrm{p}<0.05)$, and l-h OGTT plasma glucose levels $(\mathrm{r}=0.30, \mathrm{p}<0.05)$. So, $r$ value from correlation analyses for variable FPG was greater than other variables: pre-pregnancy BMI, BMI before delivery and 1-h OGTT. We also noted significant correlations between FPG with BMI before pregnancy $(\mathrm{r}=0.47, \mathrm{p}<0.01)$, BMI before delivery $(\mathrm{r}=0.47, \mathrm{p}<0.01)$, and HbAlc $(\mathrm{r}=0.25, \mathrm{p}<0.05)$.

The results from multiple regression analysis which determine independent risk factors for LGA are shown in Table 2. Gestation week of delivery and FPG were independent predictors for LGA (Beta $=0.58$ and Beta $=0.37$ respectively, $\mathrm{p}<0.01)($ Table 2$)$.

In order to evaluate fasting, 1 -h and 2 -h OGTT glucose levels for the prediction of LGA, we compared the areas under the receiver operator characteristic curve (AUC) of the ROC curves. Figure 1 shows that, for

Table 2. Influence of maternal characteristics on LGA newborns from GDM pregnancies

\begin{tabular}{lccccc}
\hline & \multicolumn{2}{c}{$\begin{array}{c}\text { Unstandardized } \\
\text { coefficients }\end{array}$} & $\begin{array}{c}\text { Standardized } \\
\text { coefficients }\end{array}$ & t & Sig. \\
\cline { 2 - 4 } & $\mathbf{B}$ & Std. Error & Beta & & \\
\hline (Constant) & -0.751 & & & & \\
pre-preg BMI & .266 & .172 & .193 & 1,543 & .128 \\
BMl before & .779 & .240 & .004 & 0.324 & .747 \\
delivery & & & & & \\
HbA1C & .778 & .595 & .016 & 1,307 & .196 \\
FPG & $\mathbf{. 2 0 6}$ & $\mathbf{. 6 4 9}$ & $\mathbf{. 3 7 7}$ & $\mathbf{3 , 1 8 5}$ & $\mathbf{. 0 0 2}$ \\
1h-OGT & .146 & .403 & .004 & .36 & .717 \\
g.w. of & $\mathbf{. 2 1 6}$ & $\mathbf{. 0 3 7}$ & $\mathbf{. 5 9 8}$ & $\mathbf{5 , 8 3 4}$ & $<. \mathbf{0 0 0 1}$ \\
delivery & & & & & \\
\hline
\end{tabular}

Multiple regression analysis model with large for gestational age as a dependent variable, according to maternal characteristics from pregnancies of women with gestational diabetes was used.

BMI: body mass index; HbA1c: glycosylated haemoglobin; FPG: fasting plasma glucose; OGT: oral glucose tolerance test; g.w.: gestational week.

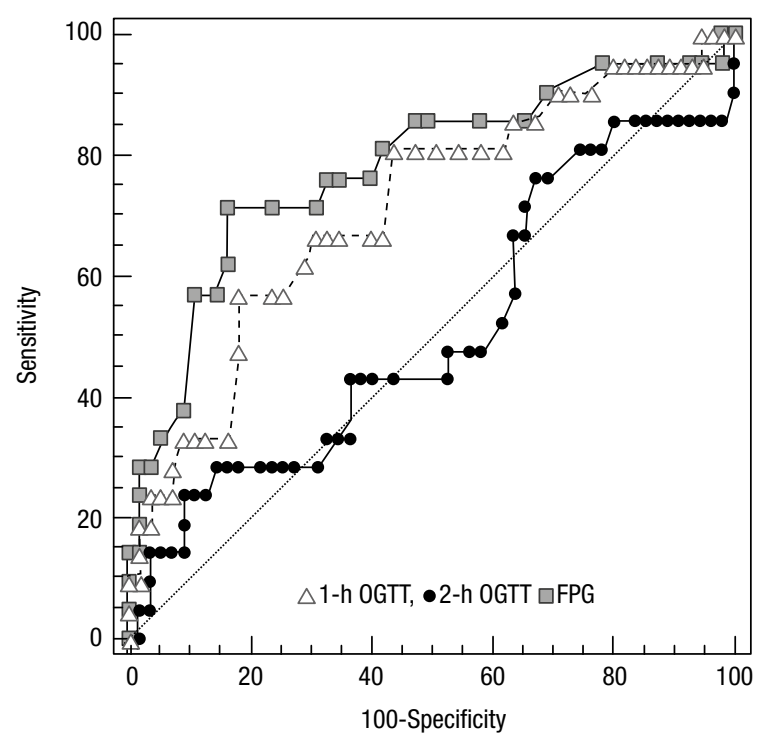

Figure 1. Receiver operator characteristics curves comparing performance of fasting, 1-h and 2-h OGT glucose levels for the prediction of LGA. The true positive rates (sensitivity) versus the false positive rates (100-specificity) are plotted for the cut off glucose levels (each data point is $0.2 \mathrm{mmol} / \mathrm{L}$ apart). OGTT: oral glucose tolerance test, FPG: fasting plasma glucose. 
LGA newborns from GDM pregnancies, fasting AUC was $0.782(0.685-0.861)$, for l-h OGTT was 0.719 (0.607-0.815), and for 2-h OGTT was 0.51 (0.3920.626). Fasting glucose levels and l-h OGTT glucose levels show statistically significant predictability for LGA ( $\mathrm{p}<0.05)$. Thus, FPG shows higher predictability than 1-h OGTT glucose levels.

\section{DISCUSSION}

The results of the study confirmed that gestational diabetes pregnancies were complicated with statistically higher percent of LGA newborns than normal pregnancies. We also showed that values at 1 -hour from 75-g OGTT have the strongest association with LGA newborns from women with GDM pregnancies.

Disproportion in the number of patients with GDM and normal pregnancies in this study is due to the implementation of the study in tertiary health institution, where obstetricians sent pregnant women with more risk factors for GDM.

It is known that fetal overgrowth is driven by a cluster of metabolic alterations associated with maternal obesity. Birth weight is largely determined by maternal factors such as prepregnancy BMI, weight gain during pregnancy and maternal serum lipid levels in mothers with GDM (14-16). We did not analyze lipid parameters, but pre-pregnancy BMI and BMI before delivery significantly correlated with LGA. In their study Charlotte and cols. concluded that maternal obesity had the strongest and independent effect on LGA in pregnancies complicated by GDM (17). According to them, incidence of LGA infants was significantly higher in obese women than in those with normal BMI, which is comparable with our findings. However, in our study we found statistically significant differences only in BMI before delivery. It is well known that FPG and OGTT glucose levels are mirror for metabolic alterations in women with GDM. FPG and 1-h OGTT glucose levels correlated and were independent risk factors for LGA. FPG will increase the risk for LGA newborns in women with GDM pregnancies especially when it is combined with higher BMI before delivery. In our study regression analysis did not show that pre-pregnancy BMI and BMI before delivery are independent predictors for LGA. This may be explained by the fact that our patients were overweight, but not obese or severely obese. Pre-pregnancy BMI and BMI before delivery were $26 \pm 6.3$ and $30.1 \pm 4.9 \mathrm{~kg} / \mathrm{m}^{2}$, respectively.
Also, this study did not find a correlation between $\mathrm{HbAlc}$ and LGA, nor was HbAlc an independent predictor for LGA. Other studies reported that the relationship between $\mathrm{HbAlc}$ and neonatal birth weight is weak $(18,19)$. It is well known that HbAlc is not an appropriate measure for the prediction for LGA babies. There is a large overlap in the distribution of HbAlc values between women with normal, borderline abnormal and mildly abnormal blood glucose values (20). Therefore, HbAlc is not a suitable test to detect mildly impaired glucose tolerance. The mean HbAlc in this study was $5.2 \pm 1.2 \%$.

Fasting plasma glucose levels and 1-h OGTT glucose levels were more powerful in prediction of LGA than 2-h OGTT glucose levels in this study. Identical with our results, Ouzilleau and cols. found that FPG levels correlated better with birth weight than the 2-h levels from 75-g OGTT in univariate, multivariate analyses, and ROC curves (21). Mello and cols. show that glucose levels at 1 -h from 75-g OGTT gave a strong association with all abnormal neonatal anthropometric characteristics including LGA (22). Perucchini and cols. recommended universal screening for GDM using a FPG of $\geq 4.8 \mathrm{mmol} / \mathrm{L}$ between 24 th and $28^{\text {th }}$ gestational week as an easier screening procedure (23). Fasting plasma glucose measurement is well tolerated and an inexpensive routine examination. So, FPG is a simple indicator for identifying high risk women with GDM. Earlier reports noted that the fasting glucose value was better than the postchallenge glucose levels in identifying LGA risk $(21,24,25)$. Similarly, Retnakaran and cols. found that FPG best predicts LGA risk, whereas post load glucose levels from OGTT predict postpartum prediabetes or diabetes risks (26). When we identify the strongly associated level of OGTT with the risk of LGA newborn, the treatment strategy for clinicians can be easy.

The investigators from HAPO study found a continuum of increasing risk of adverse outcome as each of the three (FPG, l-h, and 2-h) plasma glucose values increased (6). These adverse outcomes included LGA, cesarean delivery, neonatal hypoglycemia, and preeclampsia. In conclusion, if more than one level is exceeded, the chances for delivering LGA babies are higher. According to our results, the combination of higher FPG and 1-h values from the 75-g OGTT gives higher predictability for LGA. So, tight glucose control in these target women may be necessary to avoid LGA newborns. 
The weakness of the study is the small cluster of women and the influence of the GDM treatment on birth weight. All women were treated according to their glycemic profile with tendency to achieve fasting, postprandial and $\mathrm{HbAlc}$ values recommended for pregnant women with GDM. In spite of treatment $30 \%$ of newborns were LGA. The question remains whether we need to take into account FPG values to determine the optimal time for starting with therapy and the kind of therapy, diets or insulin. Large controlled interventional clinical trials should be done to answer this question.

In conclusion our study documented a significant association between fasting and 1-h 75-g OGTT glucose levels with LGA newborns.

Disclosure: no potential conflict of interest relevant to this article was reported.

\section{REFERENCES}

1. American College of Obstetricians and Gynecologists Committee on Practice Bulletins - Obstetrics. ACOG Practice Bulletin. Clinical management guidelines for obstetrician-gynecologists. Number 30, September 2001 (replaces Technical Bulletin Number 200, December 1994). Gestational diabetes. Obstet Gynecol. 2001;98(3):525-38.

2. Evagelidou EN, Kiortsis DN, Bairaktari ET, Giapros VI, Cholevas VK, Tzallas CS, et al. Lipid profile, glucose homeostasis, blood pressure, and obesity-anthropometric markers in macrosomic offspring of nondiabetic mothers. Diabetes Care. 2006;29(6):1197-201.

3. Snyder J, Gray-Donald K, Koski KG. Predictors of infant birth weight in gestational diabetes. Am J Clin Nutr. 1994;59(6):140914.

4. Kabali C, Werler MM. Pre-pregnant body mass index, weight gain and the risk of delivering large babies among non-diabetic mothers. Int J Gynaecol Obstet. 2007;97(2):100-4.

5. Wendland EM, Torloni MR, Falavigna M, Trujillo J, Dode MA, Campos MA, et al. Gestational diabetes and pregnancy outcomes - a systematic review of the World Health Organization (WHO) and the International Association of Diabetes in Pregnancy Study Groups (IADPSG) diagnostic criteria. BMC Pregnancy Childbirth. 2012;12:23.

6. HAPO Study Cooperative Research Group, Metzger BE, Lowe LP, Dyer AR, Trimble ER, Chaovarindr U, Coustan DR, et al. Hyperglycemia and adverse pregnancy outcomes. N Engl J Med. 2008;358(19):1991-2002.

7. International Association of Diabetes and Pregnancy Study Groups Consensus Panel, Metzger BE, Gabbe SG, Persson B, Buchanan TA, Catalano PA, Damm P, et al. International association of diabetes and pregnancy study groups recommendations on the diagnosis and classification of hyperglycemia in pregnancy. Diabetes Care. 2010;33(3):676-82.

8. Metzger BE, Coustan DR. Summary and recommendations of the Fourth International Workshop-Conference on Gestational Diabetes Mellitus. The Organizing Committee. Diabetes Care. 1998;21 Suppl 2:B161-7.

9. Black MH, Sacks DA, Xiang AH, Lawrence JM. Clinical outcomes of pregnancies complicated by mild gestational diabe- tes mellitus differ by combinations of abnormal oral glucose tolerance test values. Diabetes Care. 2010;33(12):2524-30.

10. Ferrara A, Weiss NS, Hedderson MM, Quesenberry CP Jr, Selby JV, Ergas IJ, et al. Pregnancy plasma glucose levels exceeding the American Diabetes Association thresholds, but below the National Diabetes Data Group thresholds for gestational diabetes mellitus, are related to the risk of neonatal macrosomia, hypoglycaemia and hyperbilirubinaemia. Diabetologia. 2007;50(2):298-306.

11. Caulfield $L E$, Harris $S B$, Whalen EA, Sugamori ME. Maternal nutritional status, diabetes and risk of macrosomia among Native Canadian women. Early Hum Dev. 1998;50(3):293-303.

12. Kösüs N, Kösüs $A$, Duran $M$, Turhan NO. Effect of number of abnormal oral glucose tolerance test (OGTT) values on birth weight in women with gestational diabetes. Indian J Med Res. 2013;137(1):95-101.

13. WHO Expert Committee on Diabetes Mellitus. Diabetes Mellitus. Geneva:World Health Organizarion; 1985 (Tech. Rep. Ser. no. 727).

14. Yang YD, Zhai GR, Yang HX. [Factors relevant to newborn birth weight in pregnancy complicated with abnormal glucose metabolism]. Zhonghua Fu Chan Ke Za Zhi. 2010;45(9):646-51.

15. Krstevska B, Velkoska Nakova V, Adamova G, Simeonova $\mathrm{S}$, Dimitrovski $\mathrm{Ch}$, Livrinova $\mathrm{V}$, et al. Association between foetal growth and different maternal metabolic characteristics in women with gestational diabetes mellitus. Prilozi. 2009;30(2):103-14.

16. Krstevska B, Mishevska S, Janevska E, Simeonova S, Livrinova V, Pemovska G, et al. Gestational Diabetes Mellitus - the impact of maternal body mass index and glycaemic control on baby's birth weight. Prilozi. 2009;30(2):115-24.

17. Charlotte MB, Verma A, Tucker R, Vohr BR. Metabolic syndrome in childhood: association with birth weight, maternal obesity and gestational diabetes mellitus. Pediatrics. 2005;115(3):e290-6.

18. Johnstone FD, Mao JH, Steel JM, Prescott RJ, Hume R. Factors affecting fetal weight distribution in women with type I diabetes. BJOG. 2000;107(8):1001-6.

19. Penney GC, Mair G, Pearson DW. The relationship between birth weight and maternal glycated haemoglobin (HbA1c) concentration in pregnancies complicated by Type 1 diabetes. Diabet Med. 2003;20(2):162-6.

20. Lowe LP, Metzger BE, Dyer AR, Lowe J, McCance DR, Lappin TR, et al.; HAPO Study Cooperative Research Group. Hyperglycemia and Adverse Pregnancy Outcome (HAPO) Study: associations of maternal $\mathrm{A} 1 \mathrm{C}$ and glucose with pregnancy outcomes. Diabetes Care. 2012;35(3):574-80.

21. Ouzilleau C, Roy MA, Leblanc L, Carpentier A, Maheux P. An observational study comparing 2-hour $75-g$ oral glucose tolerance with fasting plasma glucose in pregnant women: both poorly predictive of birth weight. CMAJ. 2003;168(4):403-9.

22. Mello G, Parretti E, Cioni R, Lucchetti R, Carignani L, Martini $E$, et al. The 75-gram glucose load in pregnancy: relation between glucose levels and anthropometric characteristics of infants born to women with normal glucose metabolism. Diabetes Care. 2003;26(4):1206-10.

23. Perucchini $D$, Fischer $U$, Spinas GA, Huch $R$, Huch A, Lehmann R. Using fasting plasma glucose concentrations to screen for gestational diabetes mellitus: prospective population based study. BMJ. 1999;319(7213):812-5.

24. Schaefer-Graf UM, Heuer R, Kilavuz O, Pandura A, Henrich W, Vetter K. Maternal obesity not maternal glucose values correlates best with high rates of fetal macrosomia in pregnancies complicated by gestational diabetes. J Perinat Med. 2002;30(4):313-21. 
25. Kautzky-Willer A, Bancher-Todesca D, Weitgasser R, Prikoszovich T, Steiner H, Shnawa N, al. The impact of risk factors and more stringent diagnostic criteria of gestational diabetes on outcomes in central European women. J Clin Endocrinol Metab. 2008;93(5):1689-95.
26. Retnakaran R, QiY, Sermer M, Connelly PW, Hanley AJ, Zinman B. The antepartum glucose values that predict neonatal macrosomia differ from those that predict postpartum prediabetes or diabetes: implications for the diagnostic criteria for gestational diabetes. J Clin Endocrinol Metab. 2009;94(3):840-5. 